21st Particles and Nuclei International Conference (PANIC 2017)

International Journal of Modern Physics: Conference Series

Vol. 46 (2018) 1860061 (8 pages)

(C) The Author(s)

DOI: $10.1142 / \mathrm{S} 2010194518600613$

\title{
$C P$ Violation Sensitivity at the Belle II Experiment
}

\author{
Tao Luo* (on behalf of the Belle II Collaboration) \\ Key Laboratory of Nuclear Physics \\ and Ion-beam Application (MOE) and Institute of Modern Physics, \\ Fudan University, Shanghai, China 200443 \\ luot@fudan.edu.cn
}

Published 3 May 2018

\begin{abstract}
The sensitivity on the measurements of the angles of the CKM unitarity triangle, i.e. $\phi_{1}, \phi_{2}$ and $\phi_{3}$, for Belle II experiment is presented in this letter, the CKM mechanism is expected to be tested at $1 \%$ level on Belle II.
\end{abstract}

Keywords: $C P$ Violation; the angles of the CKM unitarity triangle; the Belle II experiment.

\section{Introduction}

The weak interactions of quarks are described by the Cabibbo-KobayashiMaskawa (CKM) ${ }^{1,2}$ matrix, which needs to meet the unitary condition, so it has four degrees of freedom. If we select proper variables, the CKM matrix can be expressed by three rotation angles and one phase $(\mathrm{A}, \lambda, \rho, \eta)$. The unitary condition of CKM matrix can be converted to six free triangles in the complex plane, and one of the triangles is related to the $B$ meson decays, which is shown in Fig. 1. In this triangle, the side lengths are related to the amount of branching fractions for the corresponding decays, and also the possible $b \bar{b}$ mixing, while the widths of the three angles are related to the amount of the $C P$ Violation $(C P V)$ of difference decay processes. These angles are just what we are interested in for the following parts of this letter. Due to history reasons, these three angles have two sets of names, $\alpha$, $\beta, \gamma$, and $\phi_{1}, \phi_{2}, \phi_{3}$. They are corresponding to each other like what are shown in Fig. 1. $\phi_{1}, \phi_{2}, \phi_{3}$ are used in this letter.

The values of $\phi_{1}^{3,4,5,6}$ can be extracted from $b \rightarrow c \bar{c} s$ and $q \bar{q} s$ decay channels, such as: $B^{0} \rightarrow J / \psi K_{s}^{0}, B^{0} \rightarrow \phi K_{S}^{0}$, and $B^{0} \rightarrow \eta^{\prime} K_{S}^{0}$; the effective $\phi_{2}$ can be

*Supported by Fudan University Grant No. JIH5913023, No. IDH5913011/003.

This is an Open Access article published by World Scientific Publishing Company. It is distributed under the terms of the Creative Commons Attribution 4.0 (CC-BY) License. Further distribution of this work is permitted, provided the original work is properly cited. 


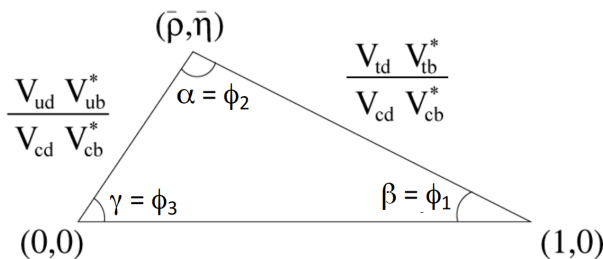

Fig. 1. The $\mathrm{B}^{0}$ Unitarity Triangle (UT).

extracted from $B \rightarrow u \bar{u} d$ processes, such as $B \rightarrow \pi \pi, \rho \rho$ and $\rho \pi$; the values of $\phi_{3}$ can be extracted from $B \rightarrow c \bar{u} s$ processes, such as the golden mode $B^{ \pm} \rightarrow D K^{ \pm}$. The expected sensitivity of the measurement of these angles from some of these decay modes on Belle II will be introduced one by one in the following parts of this letter.

\section{Time dependent measurements}

One of the most important tasks on Belle II is to measure the time dependent $C P$ violation of $B$ meson decay, from this measurement, we can extract $\phi_{1}$. This section just displays how to extract $\phi_{1}$ from $B^{0}$ decay. With asymmetric energies, $e^{+}$and $e^{-}$collide inside Belle II detector at $\Upsilon(4 \mathrm{~S})$ energy region, which is shown in Fig. 2. One $B$ meson decays to $J / \psi$ and $K_{S}$, which is taken as the signal $C P$ side; the

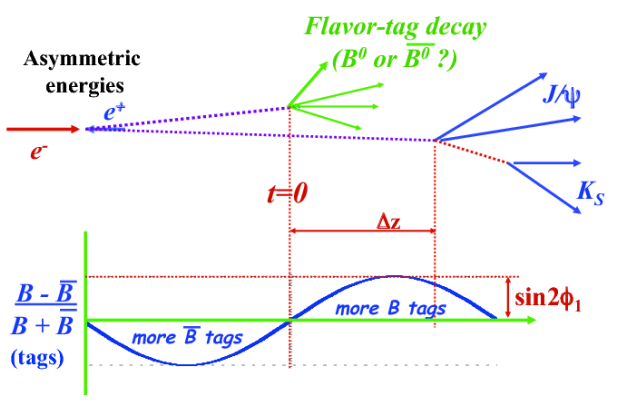

Fig. 2. Asymmetric B-factories at $\Upsilon(4 \mathrm{~S})$.

other $B$ meson decays to other final states, which is taken as the flavor-tag side; the distance between this two $B$ meson decay vertices in the boost $\operatorname{direction}(\Delta l)$ can be determined by the detector, and then converted into time difference through the equation:

$$
\Delta t \equiv t_{\text {sig }}-t_{\text {tag }}=\Delta l / \beta \gamma c .
$$

The difference of the time-dependent decay rates for the initial $B$ meson, which decays to a $C P$ eigenstate $J / \psi K_{S}$, can be described by Eq.(2); this $C P$ asymmetry parameter is related to $\mathrm{S}$ and $\mathrm{C}$, which represent the indirect and direct $C P V$. By 
fitting to the $B$ meson time dependent $C P$ asymmetry $\left(\alpha_{f_{C P}}(\Delta t)\right)$ distribution, we can extract $\mathrm{S}$ and $\phi_{1}$ from the data.

$$
\alpha_{f_{C P}}(\Delta t) \equiv \frac{\Gamma_{\bar{B} \rightarrow f_{C P}}(\Delta t)-\Gamma_{B \rightarrow f_{C P}}(\Delta t)}{\Gamma_{\bar{B} \rightarrow f_{C P}}(\Delta t)+\Gamma_{B \rightarrow f_{C P}}(\Delta t)}=\mathrm{S} \sin (\Delta \mathrm{M} \Delta \mathrm{t})-\mathrm{C} \cos (\Delta \mathrm{M} \Delta \mathrm{t}),
$$

where

$$
\mathrm{S}=-\xi_{\mathrm{f}} \sin 2 \phi_{1}, \mathrm{C} \approx 0
$$

\section{Belle II Detector on SuperKEKB}

KEKB has been upgraded to SuperKEKB, accordingly, the Belle detector has been upgraded to Belle $\mathrm{II}^{7}$. Two of the most important upgrades for the accelerator are: the beam size has been reduced to $\frac{1}{20}$ of that used for KEKB, and reaches nano meter level; the beam currents have been doubled. So totally the peaking luminosity of SuperKEKB will achieve 40 time of that for KEKB, reaching up to $8 \times 10^{35} \mathrm{~cm}^{2} \mathrm{~s}^{-1}$. The Belle II experiment expects to accumulate $50 \mathrm{ab}^{-1}$ data ${ }^{12}$ by 2024 . There are many upgrades needed for the Belle II detector in order to increase the performance and cope with much more severe background conditions. The main improvement on Belle II is in two aspects: 1 . new tracking detector: Central Drift Chamber and new vertex determination detector; 2 . two new charged particle identification detectors ${ }^{7}$.

\subsection{Vertex fit performance}

Kinematic vertex fits are usually used to identify particle decay vertex. We use benchmark for testing the Belle II vertexing performance, while the decay vertex of $J / \psi$ coming from the $B^{0} \rightarrow J / \psi K^{0}$ decay mode. The top left plot in Fig. 3 shows the fit residuals of the $z$ component of the $J / \psi$ vertex fit. A resolution of $22 \mu \mathrm{m}$ is obtained from this fit.

The same vertex fit performed using Belle Monte Carlo returns a resolution of $43 \mu \mathrm{m}$, which is two times of the one expected by Belle II, and is compatible with the expected improvement in the impact resolution due to the Belle II Pixel Vertex Detector.

From Eq. 1, the most important contribution to the $\Delta t$ resolution comes from the tagging $B$ vertex fit. For the tag side vertex fit of correctly reconstructed $B$ mesons, a resolution of $52 \mu \mathrm{m}{ }^{12}$ is obtained, which is independent of the signal $B$ decay mode. The top right plot in Fig. 3 shows the residuals of the B-tag vertex fit of fully reconstructed $B^{0} \rightarrow\left[J / \psi \rightarrow \mu^{+} \mu^{-}\right]\left[K_{s}^{0} \rightarrow \pi^{+} \pi^{-}\right]$.

In order to improve resolution of the tag side vertex fit, RAVE adaptive vertex fit algorithm has been adapted. We required that all of the tracks inputted to the vertex fit needs to have one hit on the PXD, and they are not from the signal side $B$, also and not from $K_{s}$. We constrain the fit to a region defined by an ellipsoid around the boost direction (bottom right plot in Fig. 3), where the $B$ has an higher 

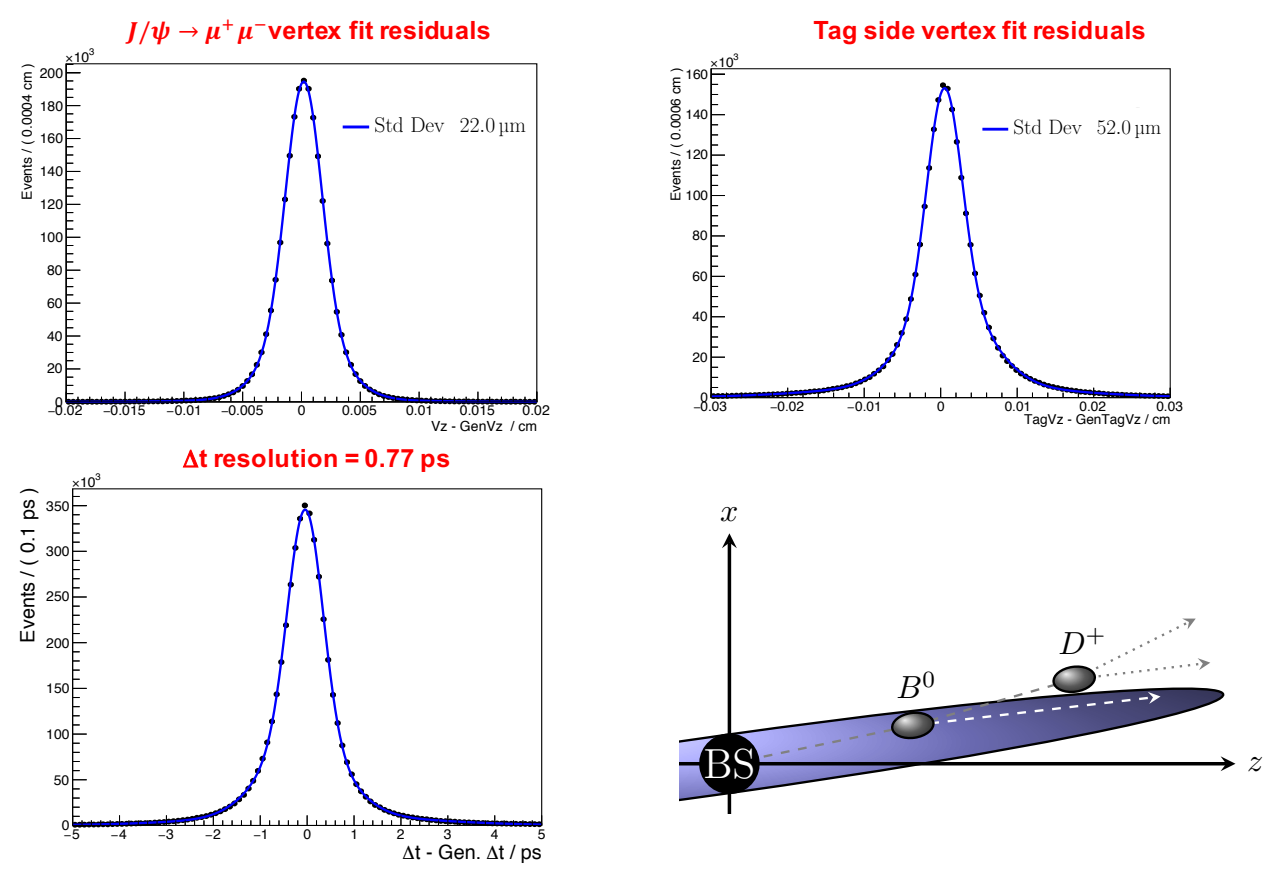

Fig. 3. Vertex fit performance of the Belle II detector.

probability to decay than a possible $D$ meson. This method helps a lot in the vertex fit.

The sensitivity to the time-dependent $\mathrm{CP}$ violating parameters of equation 2 strongly depends on the detector resolution of the $\Delta t$ distribution, which is replying on the resolution of the distance of the decay vertices of the two exclusively produced $B$ mesons (see Eq. 1). Thanks to the Belle II Pixel Vertex Detector and the new vertexing algorithms, Belle II provides an improvement, compared to Belle of the vertex resolution of both $\mathrm{B}$ mesons. The distance measurement $(\Delta l)$ translates to $\Delta t$ with a resolution of $0.77 \mathrm{ps}^{12}$, which provides a better separation capability compared to Belle. The bottom left plot in Fig. 3 shows the $\Delta t$ residuals of fully reconstructed $B^{0} \rightarrow\left[J / \psi \rightarrow \mu^{+} \mu^{-}\right]\left[K_{s}^{0} \rightarrow \pi^{+} \pi^{-}\right]$from the Belle II simulation study.

\subsection{The flavor tagging efficiency and the PID performance}

Another two key aspects of the detector performance for the CPV measurement are the flavor tagging efficiency and the PID performance. Multivariate combiner is used to improve the global performance of the flavor tagger. The FBDT combiner achieves a total effective efficiency of $35.8 \%$, the effective efficiency here reflects the fraction of real tagged events over the total events. A maximization of the effective 

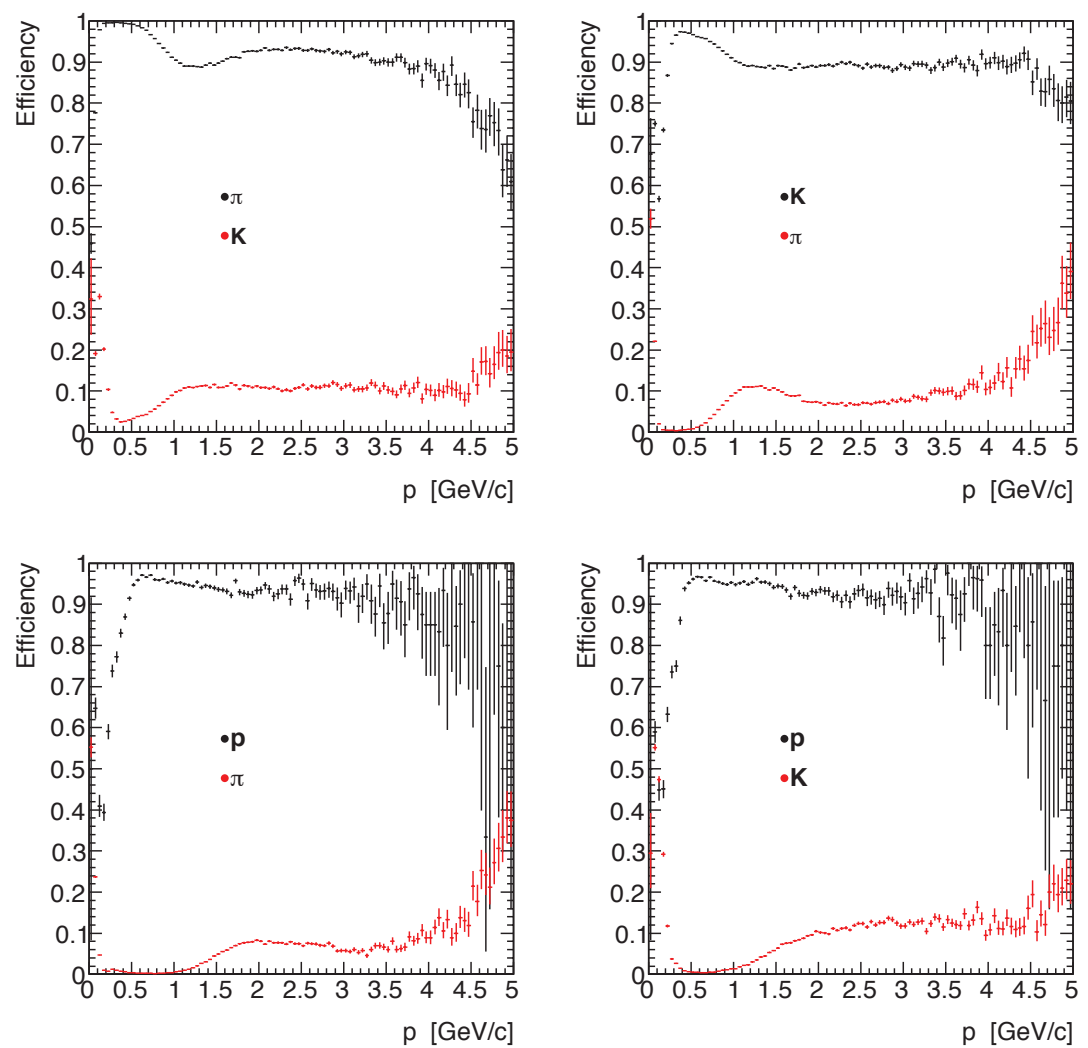

Fig. 4. Charged particle identification selection endciency for various pairs of particle types as a function of momentum in release-00-07-00 ${ }^{12}$. The black markers show the selection efficiency as determined from an inclusive MC sample without beam backgrounds, while the red markers show the fake rate. Only tracks that fall within the acceptance of at least one of the PID detectors or the CDC are considered.

efficiency results in a minimization of the statistical uncertainty for the final physical results.

Because of the new charged PID detectors, the Belle II is very powerful in the charged particle separations, especially in the low momentum region. The selection efficiency for various pairs of particle types are given in Fig. $4^{12}$.

\section{4. $\phi_{1}$ determination from $b \rightarrow c \bar{c} s$ decay channels}

As mentioned in previous section, $B^{0} \rightarrow J / \psi K^{0}$ is the golden mode for extracting $\phi_{1}$ for two reasons ${ }^{3,4}: 1$. The expected theoretical uncertainty is small; 2 . The experimental signature is clean. This process happens mainly through the tree diagram process, but there is also penguin pollution. One good thing is that the theoretical estimates on penguin pollution have been significantly improved. Table 1 shows the 
Table 1. Current status of $\phi_{1}$ determination from Belle ${ }^{8}$.

\begin{tabular}{lcccc}
\hline \hline PRL108 171802 & - & Value & stat. $\left(10^{-3}\right)$ & syst. $\left(10^{-3}\right)$ \\
\hline$J / \psi K^{0}$ & $\mathrm{~S}$ & +0.67 & 29 & 13 \\
& $\mathcal{A} \equiv-\mathrm{C}$ & -0.015 & 21 & +45 \\
\hline$c \bar{c} s$ & $\mathrm{~S}$ & +0.667 & 23 & 12 \\
& $\mathcal{A} \equiv-\mathrm{C}$ & -0.006 & 16 & 12 \\
\hline \hline
\end{tabular}

Table 2. Belle2 expected uncertainties on $\phi_{1}$ determination @ $50 \mathrm{ab}^{-1}$. Case1: irreducible syst. same as Belle; Case2: irreducible syst. (vertexing)reduced by a factor 2 due to the new Pixel Vertex detector and improved tracking and alignment algorithms ${ }^{12}$

\begin{tabular}{lcc|c|c|c}
\hline \hline Expected errors $\left(10^{-3}\right)$ & - & stat. & syst. reducible & syst.(case 1) & syst.(case 2) \\
\hline$J / \psi K^{0}$ & $\mathrm{~S}$ & 3.5 & 1.2 & 8.2 & 4.4 \\
& $\mathcal{A} \equiv-\mathrm{C}$ & 2.5 & 0.7 & +43 & +42 \\
\hline $\bar{c} s$ & $\mathrm{~S}$ & 2.7 & 2.6 & 7.0 & 3.6 \\
& $\mathcal{A} \equiv-\mathrm{C}$ & 1.9 & 1.4 & 10.6 & 8.7 \\
\hline \hline
\end{tabular}

current status of this angle determination from Belle. For S measurement, it is statistical uncertainty dominated. Table 2 shows the expected uncertainties on Belle II at $50 \mathrm{ab}^{-1}$ data, which will give results with precision better than $1 \%$ comparing to the current $5 \%$. The results will become systematic uncertainty dominated in the SuperKEKB era.

Fig. 5 shows the time dependent $C P$ asymmetry distributions that can be measured at Belle II in the $B^{0} \rightarrow J / \psi K_{s}^{0}$ and $\eta^{\prime} K_{s}^{0}$ channels with an integrated luminosity of $50 \mathrm{ab}^{-1}$. As inputs to the simulations, we set $S_{J / \psi K_{s}^{0}}=0.70$ and $S_{\eta^{\prime} K_{s}^{0}}=0.55$

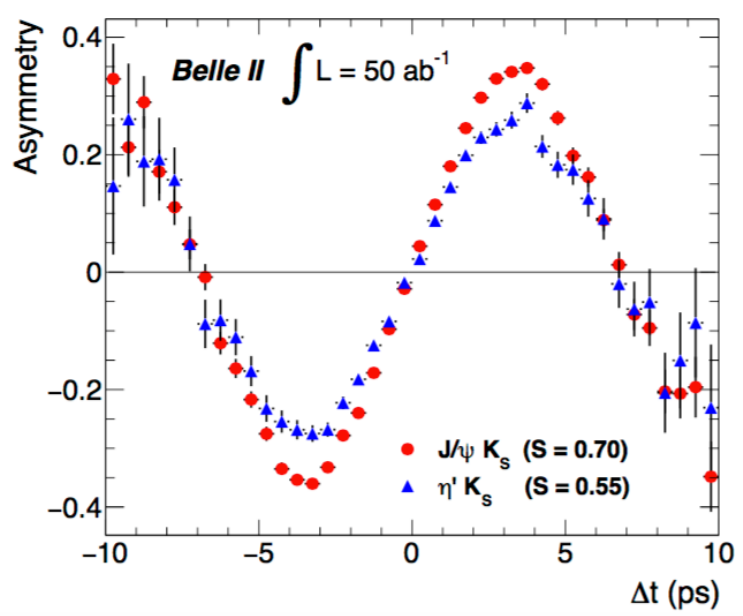

Fig. 5. Time dependent $C P$ asymmetries for the final states $J / \psi K_{s}^{0}$ (red dots) and $\eta^{\prime} K_{s}^{0}$ (blue triangles), using $S_{J / \psi K_{s}^{0}}=0.70$ and $S_{\eta^{\prime} K_{s}^{0}}=0.55$ as inputs to the Monte Carlo. With the full integrated luminosity of $50 \mathrm{ab}^{-1}$; the two values would be unambiguously distinguishable, signifying the existence of New Physics. 
(see Eq. 3). Such a difference between $S_{J / \psi K_{s}^{0}}$ and $S_{\eta^{\prime} K_{s}^{0}}$ would be an unambiguous sign of New Physics and would be easily detectable by the Belle II experiment.

\section{5. $\phi_{2}$ determination on Belle II}

The values of $\phi_{2}$ angle are determined mainly through $b \rightarrow u \bar{u} d$ processes, such as $B \rightarrow \pi \pi, \rho \rho$, and $\rho \pi$. Because of the existence of non-negligible strong phase, we can not extract $\phi_{2}$ directly but determine the effective $\phi_{2}$ like Eq. 4 . C is no longer equal to 0 , which means that there will be direct $C P$ violation.

$$
\mathrm{S}=\sin \left(2 \phi_{2}^{\mathrm{eff}}\right), \phi_{2}^{\mathrm{eff}}=\phi_{2}+\Delta \phi_{2} ; \mathrm{C} \neq 0
$$

As an example, Table. 3 shows the expected sensitivity of $\phi_{2}$ determination from $B \rightarrow \pi \pi$ channels at $50 \mathrm{ab}^{-1}$ Belle II data from the isospin analysis of MC simulation. Belle II will make big contributions on the research of these channels, especially on the branch fraction measurements.

Table 3. Branching fractions and $C P$ asymmetry parameters entering in the isospin analysis of the $B \rightarrow \pi \pi$ system: Belle measurements at $0.8 \mathrm{ab}^{-1}$ together with the expected Belle II sensitivity at $50 \mathrm{ab}^{-1} .{ }^{12}$

\begin{tabular}{cccc}
\hline \hline & Value & $0.8 \mathrm{ab}^{-1}$ & $50 \mathrm{ab}^{-1}$ \\
\hline $\mathcal{B}_{\pi^{+} \pi^{-}}\left[10^{-6}\right]$ & 5.04 & $\pm 0.21 \pm 0.18^{10}$ & $\pm 0.03 \pm 0.08$ \\
$\mathcal{B}_{\pi^{\prime} \pi^{\prime}}\left[10^{-6}\right]$ & 1.31 & $\pm 0.19 \pm 0.18^{9}$ & $\pm 0.04 \pm 0.04$ \\
$\mathcal{B}_{\pi^{+} \pi^{\prime}}\left[10^{-6}\right]$ & 5.86 & $\pm 0.26 \pm 0.38^{10}$ & $\pm 0.03 \pm 0.09$ \\
$\mathrm{C}_{\pi^{+} \pi^{-}}$ & -0.33 & $\pm 0.06 \pm 0.03^{11}$ & $\pm 0.01 \pm 0.03$ \\
$\mathrm{~S}_{\pi^{+} \pi^{-}}$ & -0.64 & $\pm 0.08 \pm 0.03^{11}$ & $\pm 0.01 \pm 0.01$ \\
$\mathrm{C}_{\pi^{0} \pi^{0}}$ & -0.14 & $\pm 0.36 \pm 0.12^{9}$ & $\pm 0.03 \pm 0.01$ \\
$\mathrm{~S}_{\pi^{0} \pi^{0}}$ & - & - & $\pm 0.29 \pm 0.03$ \\
\hline \hline
\end{tabular}

\section{Summary}

Belle has been a successful $B$ factory, especially for the research on $C P V$. Major upgrades of KEKB and Belle have been made to build SuperKEKB and Belle II. CKM mechanism will be tested at $1 \%$ level ${ }^{12}$ on Belle II. Some flavor variables are still to be measured precisely, therefore a lot of room for discoveries at Belle II are expected!

\section{References}

1. N. Cabibbo, Phys. Rev. Lett. 10, 531 (1963).

2. M. Kobayashi and T. Maskawa, Prog. Th. Phys. 49652 (1973).

3. K. Abe et al. (Belle Collaboration) Phys. Rev. Lett. 87, 091802 (2001).

4. B. Aubert et al. (BABAR Collaboration) Phys. Rev. Lett. 87, 241801 (2001).

5. K. Abe et al. (Belle Collaboration) Phys. Rev. D 66, 071102 (2002).

6. B. Aubert et al. (BABAR Collaboration) Phys. Rev. Lett. 89, 201802 (2002).

7. T. Abe, Belle II Collaboration (2010), arXiv:1011.0352. 
8. C. Patrignani et al. (Particle Data Group), Chin. Phys. C, 40, 100001 (2016) and 2017 update (2017).

9. arXiv:1705.02083.

10. Y.-T. Duh et al. (Belle Collaboration) Phys. Rev. D 87, 031103 (2013).

11. J. Dalseno et al. (Belle Collaboration) Phys. Rev. D 88, 092003 (2013).

12. The Belle II collaboration and B2TiP theory community, The Belle II Physics Book, to be published. 